\title{
Parametric tests for partly interval-censored failure time data under Weibull distribution via multiple imputation
}

\begin{abstract}
The statistical problem considered in this article is the parametric treatment comparison when partly interval-censored failure time data exist. Partly interval-censored failure time data are composed of exact observations and interval-censored observations. This phenomenon often occurs in clinical trials and health studies that require periodic following up with patients. The authors constructed a score test and likelihood ratio test for this type of failure time data under Weibull distributions using multiple imputation technique. A simulation study and a modified secondary data set from breast cancer study are used to assess the proposed test and illustrate the differences between the two tests. The results indicate that the presented procedure works well for both tests, but the likelihood ratio test is better than the score test in certain situations.
\end{abstract}

Keyword: Breast cancer study; Likelihood ratio test; Multiple imputation; Partly intervalcensored data; Score test; Weibull distribution. 